
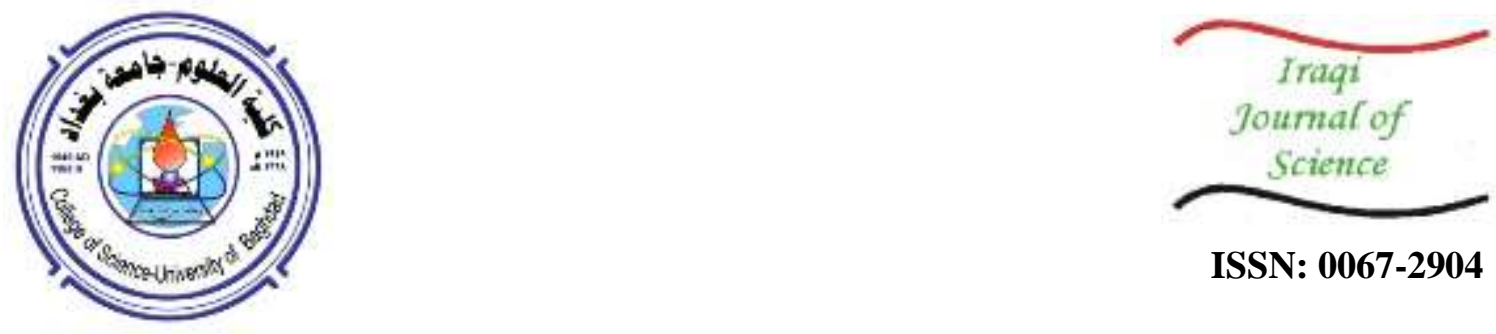

ISSN: 0067-2904

\title{
Dependent Element and Free Actions of Centralizer and Reverse Centralizer on Prime and Semiprime Semirings
}

\author{
Maryam K. Rasheed*, Abdulrahman H. Majeed \\ Department of Mathematics, College of Science, University of Baghdad, Baghdad, Iraq
}

\begin{abstract}
This paper develops the work of Mary Florence et.al. on centralizer of semiprime semirings and presents reverse centralizer of semirings with several propositions and lemmas. Also introduces the notion of dependent element and free actions on semirings with some results of free action of centralizer and reverse centralizer on semiprime semirings and some another mappings.
\end{abstract}

Keywords: - Prime semiring, Semiprime semiring, Dependent element, Free actions, Centralizer, Reverse centralizer.
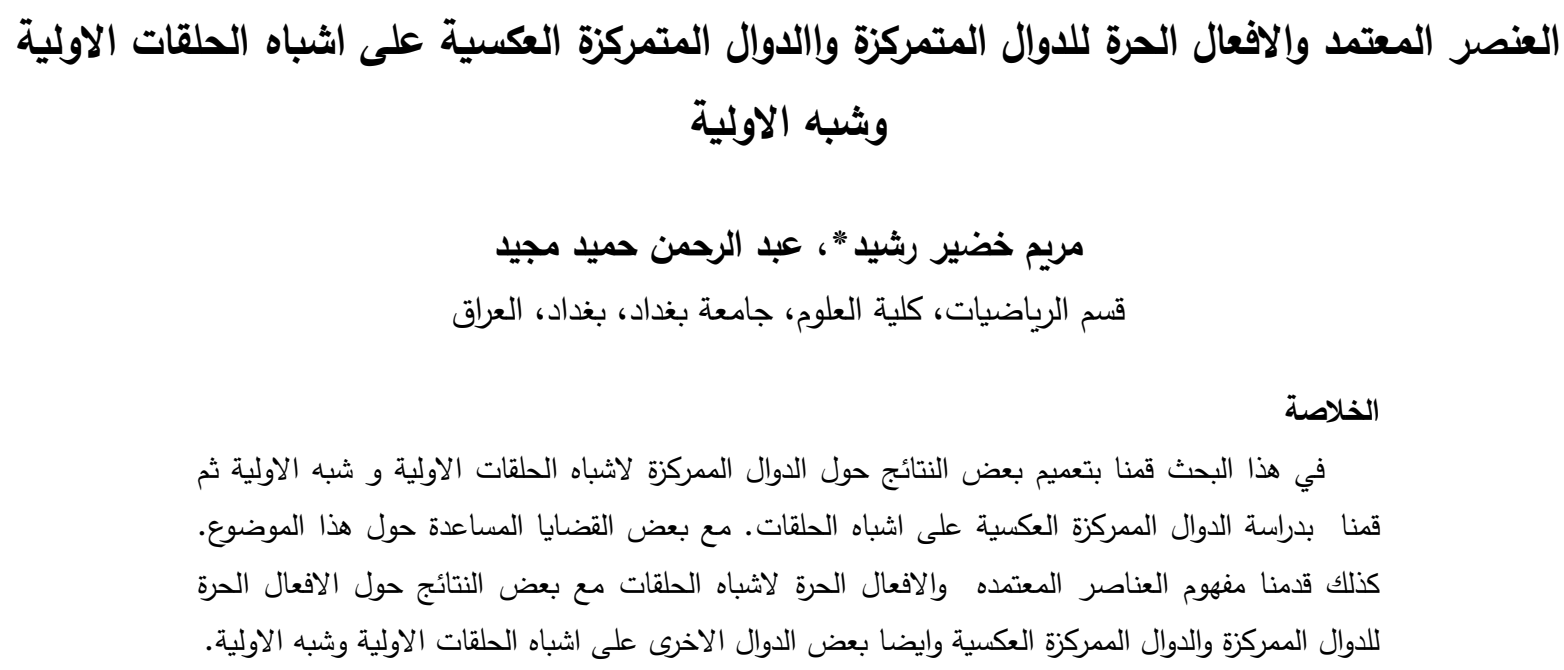

\section{Introduction}

The notion of semirings was defined by Vandiver in 1934 [1]. Then Jonathan S. Golan discussed semirings and their applications with illustrative various examples [2]. Thereafter many researches interested in the study of semirings, it's properties and defined different types of maps on it. Chandramouleeswaran and Thiruveni $[3,4]$ worked on diverse kinds of derivations. Then Mary Florence reduced the work of Zalar and Vukman [5 - 7] on rings and present centralizer on semiprime semirings and some relation related to it in $[8,9]$.

Murray and Von Neumann $[10,11]$ introduce free action concept by using dependent element on abelian algebra, after this kallman [12] generalized the notion of free action on algebra not necessary 
abelian. Then many researches extend the work of dependent element and free action on many types of mappings [13 - 15].

A nonempty set $S$ with two binary operation + and - is said to be a semiring if and only if $(S,+)$ is semigroup, $(S, \cdot)$ is semigroup and multiplication distributive with respect to addition. We called $S$ commutative if and only if $x \cdot y=y \cdot x$ for all $x, y \in S$. Also $S$ is called additively cancellative if $x+y=x+z$ implies $y=z$ for all $x, y, z \in S$, and it is called multiplicatively cancellative if $x . y=x . z$ implies $y=z$ for all $x, y, z \in S$, thus $S$ is called cancellative semiring if and only if it is both additively cancellative and multiplicatively cancellative [2]. The center of $S$ defined as $Z(S)=$ $\{x \in S: x \cdot y=y \cdot x$ for all $y \in S\}[8]$.

A semiring $S$ is called prime if whenever $x S y=0$ implies either $x=0$ or $y=0$ for all $x, y \in S$ and it is called semiprime if whenever $x S x=0$ implies $x=0$ for all $x \in S$ [3].

An additive map $d: S \rightarrow S$ is called derivation on $S$ if $d(x y)=d(x) y+x d(y)$ for all $x, y \in S$. An additive map $T: S \rightarrow S$ is called left (right) centralizer if $T(x y)=T(x) y(T(x y)=x T(y))$ for all $\mathrm{x}, \mathrm{y} \in \mathrm{S}$. If $\mathrm{T}$ is both left and right centralizer then it is called centralizer of $\mathrm{S}$ [8].

This research extend some results of centralizer on prime and semiprime semirings and study reverse centralizer of semirings with some results on prime and semiprime semirings. Also present dependent element on semiring and free action concept with some important results of free action on centralizer, reverse centralizer and another mappings on semiprime semirings.

\section{Centralizer and Reverse Centralizer on Prime and Semiprime Semirings}

Definition2.1: - Let $S$ be a semiring, an additive map $T: S \rightarrow S$ is called left (right) reverse centralizer if $T(x y)=T(y) x(T(x y)=y T(x))$ for all $\mathrm{x}, \mathrm{y} \in \mathrm{S}$. A reverse centralizer of $\mathrm{S}$ is both left and right reverse centralizer.

Lemma2.2: - Let $S$ be a prime semiring and I be a nonzero ideal of S. Let $T: S \rightarrow S$ be a left (right) centralizer. If $T=0$ on I, then $T=0$ on S.

Proof: - Suppose that $T=0$ on I, let $0 \neq \mathrm{u} \in \mathrm{I}$ and $0 \neq \mathrm{r} \in \mathrm{S}$. Then $T(r u)=T(r) u=0$.

Replace $\mathrm{u}$ by $\mathrm{s} \mathrm{u}$, where $\mathrm{s} \in \mathrm{S}$, then $T(r) s u=0$ for all $\mathrm{u} \in \mathrm{I}$ and $\mathrm{r}, \mathrm{s} \in \mathrm{S}$.

$T(r) S u=0$ for all $\mathrm{u} \in \mathrm{I}$ and $\mathrm{r} \in \mathrm{S}$. By primness of $\mathrm{S}$ and since $\mathrm{u} \neq 0$ we get $T(r)=0$ for all $\mathrm{r} \in \mathrm{S}$. Then $\mathrm{T}=0$ on $\mathrm{S}$.

Lemma2.3: - Let $\mathrm{S}$ be a prime semiring and I be a nonzero ideal of $\mathrm{S}$. Let $T: S \rightarrow S$ be a left (right) reverse centralizer, if $T=0$ on $\mathrm{I}$, then $T=0$ on $\mathrm{S}$.

Proof: - Similar as above.

Theorem2.4: - Let $\mathrm{S}$ be a cancellative prime semiring and I be a nonzero ideal of S. Let $T: S \rightarrow S$ is left (right) centralizer on I, if $T(u v)=T(v u)$ for all $\mathrm{u}, \mathrm{v} \in \mathrm{I}$, then $\mathrm{S}$ is commutative.

Proof: - Take T is left centralizer of $\mathrm{I}$, then

$$
\begin{aligned}
& T(u v)=T(u) v \text { for all } \mathrm{u}, \mathrm{v} \in \mathrm{I} \\
& T(u v)=T(v u) \text { for all } \mathrm{u}, \mathrm{v} \in \mathrm{I}
\end{aligned}
$$

From (1) and (2) we have, $T(v u)=T(u) v$ for all $u, v \in I$.

Replace $\mathrm{u}$ by u w, where $\mathrm{w} \in \mathrm{I}$,

$$
\begin{aligned}
T(u w) v & =T(v u w) \\
T(u) w v & =T(v u) w \\
& =T(u v) w \\
& =T(u) v w
\end{aligned}
$$

Since $\mathrm{S}$ is cancellative we have, $w v=v w$ for all $v, w \in I$.

Then I is commutative, by Lemma 2.22 [16] we get $S$ is commutative.

Similarly proved when $\mathrm{T}$ is right centralizer.

Theorem2.5: - Let $S$ be a prime semiring and I be a nonzero ideal of S. Let $T: S \rightarrow S$ be a left (right) reverse centralizer on $\mathrm{I}$, if $T(u v)=T(v u)$ for all $\mathrm{u}, \mathrm{v} \in \mathrm{I}$, then $S$ is commutative.

Proof: - Similar as above.

Theorem2.6: - Let $S$ be a semiprime semiring and I be a nonzero ideal of S. Let T: $\mathrm{S} \rightarrow \mathrm{S}$ be a left (right) centralizer on I, if $T(u) T(v)=0$ for all $\mathrm{u}, \mathrm{v} \in \mathrm{I}$, then $T=0$ on S.

Proof: - let $\mathrm{T}(\mathrm{u}) \mathrm{T}(\mathrm{v})=0$ for all $\mathrm{u}, \mathrm{v} \in \mathrm{I}$.

Replace u by v s, where s $\in \mathrm{S}$, then $T(v s) T(v)=T(v) s T(v)=0$ for all $\mathrm{s} \in \mathrm{S}$.

Hence $T(v) S T(v)=0$.By semiprimness of $S$ we get, $T(v)=0$ for all $\mathrm{v} \in \mathrm{I}$. 
Then $\mathrm{T}=0$ on $\mathrm{I}$ and by Lemma 2.3 we get $T=0$ on $\mathrm{S}$.

Similarly proved when $\mathrm{T}$ is right centralizer.

Theorem2.7: - Let $\mathrm{S}$ be a semiprime semiring and I be a nonzero ideal of S. Let $T: S \rightarrow S$ is left (right) reverse centralizer on I, if $T(u) T(v)=0$ for all $\mathrm{u}, \mathrm{v} \in \mathrm{I}$, then $T=0$ on $\mathrm{S}$.

Proof: - Similar as above.

\section{Dependent Element and Free Actions on Prime and Semiprime Semirings}

Definition3.1: - Let $\mathrm{S}$ be a semiring, $f: S \rightarrow S$ be any mapping and a $\in \mathrm{S}$. If $f(x) a=a x$ holds for all $\mathrm{x} \in \mathrm{S}$ then $a$ is called dependent element of $f$. The set of all dependent element of $\mathrm{f}$ denoted by $D_{p}(f)$.

Theorem3.2: - Let $\mathrm{S}$ be a cancellative semiring and $\mathrm{T}$ be a left (right) centralizer of $\mathrm{S}$. Let a $\in \mathrm{S}$, then $\mathrm{a} \in D_{p}(T)$ if and only if $\mathrm{a} \in Z(S)$ and $T(a)=a$.

Proof: - Let $\mathrm{a} \in D_{p}(T)$ then $T(x) a=a x$ for all $\mathrm{x} \in \mathrm{S}$, replace $\mathrm{x}$ by $x y$, where $\mathrm{y} \in \mathrm{S}$ then,

$$
\begin{aligned}
T(x y) a=T(x) y a & =a x y \\
& =T(x) a y
\end{aligned}
$$

Since $\mathrm{S}$ is cancellative we get, $y a=a y$ for all $\mathrm{y} \in \mathrm{S}$, then $a \in Z(S)$.

Thus $T(a y)=T(a) y=T(y) a=T(y a)$.

Then $T(a) y=a y$, since $\mathrm{S}$ is cancellative then $T(a)=a$.

Conversely, we have $a \in Z(S)$ with $T(a)=a$, now replace a by $\mathrm{x}$ a, where $\mathrm{x} \in \mathrm{S}$, then

$T(x a)=T(x) a=x a$ for all $\mathrm{x} \in \mathrm{S}$. Then $a \in D_{p}(T)$.

Similarly proved when $\mathrm{T}$ is right centralizer.

Theorem3.3: - Let $\mathrm{S}$ be a semiprime semiring and $\mathrm{T}$ be a left (right) reverse centralizer of $\mathrm{S}$. Let a $\in \mathrm{S}$ then a $\in D_{p}(T)$ if and only if $a \in Z(S)$ and $T(a)=a$.

Proof: - Let a $\in D_{p}(T)$ then $\mathrm{T}(\mathrm{x}) \mathrm{a}=\mathrm{a} \mathrm{x}$ for all $\mathrm{x} \in \mathrm{S}$.

Multiply both sides from the left by a $\mathrm{y}$, where $\mathrm{y} \in \mathrm{S}$ then,

$$
\begin{aligned}
a x a y & =T(x) a a y \\
& =T(a x) a y \\
& =T(a y a x) \\
& =T(y a x) a \\
& =T(x) a y a .
\end{aligned}
$$

We have $T(x) a[a, y]=0$ for all $\mathrm{x}, \mathrm{y} \in \mathrm{S}$, then

Replace $\mathrm{x}$ in (1) by y $\mathrm{x}$, we get

$$
a x[a, y]=0 \text { for all } x, y \in S
$$

Multiply (1) from the left by y, we have

$$
\text { a } y x[a, y]=0 \text { for all } x, y \in S
$$

$$
\text { y a } x[a, y]=0 \text { for all } x, y \in S
$$

From (2) and (3) we get, $[a, y] x[a, y]=0$ for all $\mathrm{x}, \mathrm{y} \in \mathrm{S}$.

Then $[a, y] S[a, y]=0$ for all y $\in \mathrm{S}$. By semiprimness we get $a \in Z(S)$.

Thus, $T(a y)=T(y a)=T(a) y=a y$.

Then $(T(a)-a) y=0$. By semiprimness we get $T(a)=a$.

Conversely, we have $a \in Z(S)$ with $T(a)=a$.

Replace a by a $\mathrm{x}$, where $\mathrm{x} \in \mathrm{S}$, then $T(a x)=T(x) a=x a$ for all $\mathrm{x} \in \mathrm{S}$, then a $\in D_{p}(T)$.

Similarly proved when $\mathrm{T}$ is right reverse centralizer.

Lemma3.4: - Let $\mathrm{S}$ be a semiring and $\mathrm{T}$ be a left (right) reverse centralizer of $\mathrm{S}$. Then $D_{p}(T)$ is subsemiring of $Z(S)$.

Proof: - Let a, $\mathrm{b} \in D_{p}(T)$, to prove $a+b$ and $a . b \in D_{p}(T)$.

By Theorem 3.3 we have $a, b \in Z(S), T(a)=a$ and $T(b)=b$.

It's clear that $a+b$ and $a \cdot b \in Z(S)$.

Now since $\mathrm{T}$ is left centralizer then,

$T(a+b)=T(a)+T(b)=a+b$ and $T(a b)=T(a) b=a b$.

By Theorem 3.3 we have $\mathrm{a}+\mathrm{b}$ and $\mathrm{a} \mathrm{b} \in D_{p}(T)$, then $D_{p}(T)$ is subsemiring of $\mathrm{Z}(\mathrm{S})$.

Lemma3.5: - Let $\mathrm{S}$ be a semiring and $\mathrm{T}$ be a left (right) reverse centralizer of S. $D_{p}(T)$ is subsemiring of $Z(S)$.

Proof: - Similar as above. 
Lemma3.6: - Let $\mathrm{S}$ be a commutative semiring and $\mathrm{T}$ be a left (right) centralizer of $\mathrm{S}$, then $D_{p}(T)$ is an ideal of $\mathrm{S}$.

Proof: - Since $D_{p}(T)$ is subsemiring of $Z(S)$, we need only to show that as $\in D_{p}(T)$ for some $s \in S$.

$T(x) a s=a x s=a s x$, for all $\mathrm{x} \in \mathrm{S}$. Then $D_{p}(T)$ is an ideal of $\mathrm{S}$.

Lemma3.7: - Let $\mathrm{S}$ be a semiring and $\mathrm{T}$ be a left (right) reverse centralizer of $\mathrm{S}$, then $D_{p}(T)$ is an ideal of $\mathrm{S}$.

Proof: - Since $D_{p}(T)$ is subsemiring of $Z(S)$, we need only to show that a s $\in D_{p}(T)$ for some s $\in \mathrm{S}$. $T(x) a s=T(a s x)=T(s x a)=T(a) s x=a s x$ for all $\mathrm{x} \in \mathrm{S}$. Then $D_{p}(T)$ is an ideal of $\mathrm{S}$.

Definition3.8: - Let $S$ be a semiring, a mapping $f: S \rightarrow S$ is called free action if zero is the only dependent element of $\mathrm{f}$.

Theorem3.9: - Let $\mathrm{S}$ be a prime semiring, I be a nonzero ideal of $\mathrm{S}$. Suppose that $T: S \rightarrow S$ is left (right) centralizer of $\mathrm{S}$ such that $\mathrm{T}$ not identity map on $\mathrm{I}$, then $\mathrm{T}$ is free action.

Proof: - Let a $\in D_{p}(T)$ then $T(x) a=a x$ for all $\mathrm{x} \in \mathrm{S}$.

Replace $\mathrm{x}$ by $\mathrm{u} \mathrm{x}$, where $\mathrm{u} \in \mathrm{I}$, then $T(u x) a=T(u) x a=a u x$ for all $\mathrm{u} \in \mathrm{I}$ and $\mathrm{x} \in \mathrm{S}$.

By Theorem 3.3 we have $a \in Z(S)$, then $\mathrm{T}(\mathrm{u}) \mathrm{x} \mathrm{a}=\mathrm{u} \times \mathrm{a}$.

$(T(u)-u) \times a=0$ for all $\mathrm{u} \in \mathrm{I}, \mathrm{x} \in \mathrm{S}$.

By primness and since $\mathrm{T}$ is not identity map on $\mathrm{I}$ we get $\mathrm{a}=0$, then $\mathrm{T}$ is free action.

Theorem3.10: - Let $S$ be a prime semiring, I be a nonzero ideal of S. Suppose that $T: S \rightarrow S$ is left (right) reverse centralizer of $\mathrm{S}$ such that $\mathrm{T}$ not identity map on $\mathrm{I}$, then $\mathrm{T}$ is free action.

Proof: - Similar as above.

Lemma3.11: - Let $S$ be a semiprime semiring, then $S$ has no nonzero nilpotent element.

Proof: - Trivial.

Theorem3.12: - Let $\mathrm{S}$ be a 2_torsion free prime semiring and $T: S \rightarrow S$ is left (right) centralizer of $\mathrm{S}$. Then a mapping $\Phi$ on $\mathrm{S}$ which is defined by $\Phi(x)=[T(x), x]$ for all $\mathrm{x} \in \mathrm{S}$, is free action.

Proof: - Let $a \in D_{p}(\Phi)$, then $\Phi(x) a=[T(x), x] a=a x$ for all a $\in \mathrm{S}$.

By linearization above equation we get,

Replace $\mathrm{y}$ in above equation by a y we have,

$$
\begin{gathered}
([T(x), x]+[T(x), y]+[T(y), x]+[T(y), y])=a x+a y . \\
{[T(x), y] a+[T(y), x] a=0 \text { for all } \mathrm{x}, \mathrm{y} \in \mathrm{S}}
\end{gathered}
$$

$$
\begin{gathered}
{[T(x), y a] a+[T(y a), x] a=[T(x), a y] a+[T(y) a, x] a=0} \\
a[T(x), y] a+[T(x), a] y a+T(y)[a, x] a+[T(y), x] a a=0 \\
a[T(x), y] a+[T(y), x] a a=a^{2}([T(x), y]+[T(y), x])=0
\end{gathered}
$$

Put $y=x$ in above equation we get, $2 a^{2}(\Phi(x))=a^{2}(\Phi(x))=0$ for all $\mathrm{x} \in \mathrm{S}$.

By primness and since $\Phi$ is nonzero we have $a^{2}=0$.

Then by Lemma $3.11 \mathrm{a}=0$, hence $\Phi$ is free action.

Theorem3.13: - Let $S$ be a 2-torsion free prime semiring and $T: S \rightarrow S$ is left (right) reverse centralizer of S. Then a mapping $\Phi$ on $\mathrm{S}$ which is defined by $\Phi(x)=[T(x), x]$ for all $\mathrm{x} \in \mathrm{S}$, is free action.

Proof: - Similar as above.

Theorem3.14: - Let $S$ be a semiprime semiring and $d: S \rightarrow S$ is derivation. Then $\mathrm{d}$ is free action.

Proof: - Let $a \in D_{p}(d)$, then $d(x) a=a x$ for all $\mathrm{x} \in \mathrm{S}$, replace $\mathrm{x}$ by a $\mathrm{x}$ we have,

$$
\begin{gathered}
d(a x) a=d(a) x a+a d(x) a=a a x \\
d(a) x a=0 \text { for all } x \in S
\end{gathered}
$$

By primness and since $\mathrm{d}$ is nonzero derivation, we get $\mathrm{a}=0$ then $\mathrm{d}$ is free action.

Theorem3.15: - Let $S$ be a semiprime semiring and $T: S \rightarrow S$ be a centralizer. Suppose that $\mathrm{d}$ is derivation of $\mathrm{S}$. then $\Psi=d \circ T$ is free action.

Proof: - Let $a \in D_{p}(\Psi)$, then $\Psi(x) a=a x$ for all $\mathrm{x} \in \mathrm{S}$.

$$
\begin{gathered}
d \circ T(x) a=a x \text { for all } x \in S \\
d(T(x)) a=a x
\end{gathered}
$$

Replace $\mathrm{x}$ by $\mathrm{x} y$, where $\mathrm{y} \in \mathrm{S}$, implies

$$
\begin{aligned}
& d(T(x y)) a=d(T(x) y) a=a x y \\
& d(T(x)) y a+T(x) d(y) a=a x y
\end{aligned}
$$




$$
\begin{gathered}
d \circ T(x) y a+T(x) d(y) a=a x y \\
T(x) d(y) a=d \circ T(x)[a, y] \text { for all } x, y \in S
\end{gathered}
$$

Replace y by a y in (2) we have,

$$
\begin{gathered}
T(x) d(a y) a=d \circ T(x)[a, a y]=d \circ T(x) a[a, y] \\
T(x) d(a) y a+T(x) a d(y) a=a x[a, y]
\end{gathered}
$$

Multiply (3) by z on the left, where $\mathrm{z} \in \mathrm{S}$, we get,

$$
z T(x) d(a) y a+z T(x) a d(y) a=z a x[a, y]
$$

Replace $\mathrm{x}$ by $\mathrm{z} \mathrm{x}$ in (3) we get,

$$
T(z x) d(a) y a+T(z x) a d(y) a=a z x[a, y]
$$

From (4) and (5) we have,

$$
[a, z] x[a, y]=0 \text { for all } \mathrm{x}, \mathrm{y}, \mathrm{z} \in \mathrm{S}
$$

Put $z=y$ in above equation implies,

$$
[a, y] x[a, y]=0 \text { for all } \mathrm{x} \in \mathrm{S}
$$

By semiprimness we get, $[a, y]=0$ for all $\mathrm{y} \in \mathrm{S}$.

Thus a $\in \mathrm{Z}(\mathrm{S})$. From (2) we have,

$$
T(x) d(y) a=0 \text { for all } x, y \in S
$$

Now replace y by $\mathrm{T}(\mathrm{y})$ in (6) and then using (1) we get,

$$
T(x) a=0 \text { for all } \mathrm{x} \in \mathrm{S}
$$

Take $d(T(x) a)=d(0)=0$ we obtain, $d \circ T(x) a+T(x) d(a)=0$

Multiply above equation by a on the right,

$$
d \circ T(x) a^{2}+T(x) d(a) a=0
$$

Now using (6) we get $d \circ T(x) a^{2}=0$ and from (1) we get, $a x a=0$ for all $\mathrm{x} \in \mathrm{S}$.

By semiprimness of S we get $a=0$ then $\Psi$ is free action.

\section{References}

1. Vandiver H. S. 1934. Note on simple type of algebra in which the cancellation law of addition does not hold, Math. Soc., 40: 916- 920.

2. Jonathan S. Golan. 1992. Semirings and their applications, University of Haifa, Haifa, Israel.

3. Chandramouleeswaran M., Thiruveni V. 2010. On Derivations of Semirings, Advances in Algebra, 3(1): $123-131$.

4. Chandramouleeswaran M., Thiruveni V. 2011. A Note on $\alpha$ Derivations in Semirings, International Journal of pure and applied Sciences and Technology, 2(1): 71-77.

5. Zalar B..1991. on Centralizer of Semiprime Rings, Commentationes Mathematica Universitatis Carolinae, 23(4): 609 - 614.

6. Joso Vukman, 2001. Centralizer on Semiprime Rings, Comment. Math. Univ. Carolinae, 42(2): $237-245$.

7. Joso Vukman, 2005. On Dependent Element and related problems in Rings, international Mathematical Journal, 6(2): 93 - 112.

8. Mary D., Murugesan R., Namasivayam P. 2016. Centralizers on Semiprime Semirings, IOSR Journal of Mathematics, 12(3): 86 - 93.

9. Mary D., Murugesan R., Namasivayam P. 2017. Some Relations Related to Centralizers on Semiprime Semirings, Annals of Pure and Applied Mathematics, 13(1): 119 - 124.

10. Murray F. J., Von Neumann J. 1936. on Rings of Operators, Ann. Math., 37(1): 116 -229.

11. Von Neumann J. 1940. On Rings of Operators III, Ann. Math., 41: 94 - 161.

12. Kallman R. 1968. A Generalizations of Free Actions, Duke Math. Journal, 36: 781-789.

13. Samman M. S. 2008. Dependent Element of left Centralizer of Semiprime Rings, Arabian Journal of Science and Engineering, 33(2): 313 - 317.

14. Laradji A. and Thaheem A. B. . 1998. On Dependent Element of Semiprime Rings, Math. Japon, 47(1): $29-31$.

15. Hentzel, I. R., Daif M. N., Sayed Tammam M., Claus Haetinger. 2011. On Free Action and Dependent Elements in Rings, Algebra and its Apllications, 70 - 83.

16. Rasheed, M. K. and Majeed, A. H. 2019. Some Results of $(\alpha, \beta)$ Derivation on prime semiring, Iraqi Journal of Science, 60(5): 1154-1160. 